\title{
The Museum of Consciousness: Interactive, audio-based exhibits for cultivating altered states of consciousness
}

\author{
Carl Hayden Smith \\ Ravensbourne University \\ London, United Kingdom \\ c.smith@rave.ac.uk
}

\author{
Alex Zhao \\ University of Pennsylvania \\ Philadelphia, USA \\ alexzhao@seas.upenn.edu
}

\author{
Kenneth Shinozuka \\ University of Oxford \\ Oxford, United Kingdom \\ kenneth.shinozuka@psych.ox.ac.uk
}

\author{
Michael Ronen \\ Wonderland Immersive Design \\ Los Angeles, USA \\ themichaelronen@gmail.com
}

\begin{abstract}
The Museum of Consciousness contains exhibits by a multitude of artists, mavericks and consciousness explorers who are here to transmit their most treasured work: For the first exhibition we decided to use short, audio-based samples that are designed to cultivate and manufacture expansive states of consciousness. The range of samples encompassed by the current iteration of the museum includes mindfulness meditations, "placebo highs," sound journeys, novel modes of introspection, and more. The structure of the museum is centered around recipes for consciousness augmentation. The museum also has an interactive component: visitors will rate the samples according to Raph Milliere's 6D model of self-consciousness, which was inspired by the effects of psychedelics and meditation on subjective experience. Additionally, visitors will play a game in which they try to figure out the ratings that the curators had pre-assigned to the samples. Finally, visitors will have the opportunity to provide feedback to the artists, as part of the cyclically regenerative system at the heart of the museum.
\end{abstract}

Consciousness. Altered states of consciousness. Meditation. Psychedelics. Recipes for consciousness. Augmentation. Consciousness stacking.

\section{INTRODUCTION}

How do we map out the range of conscious experiences that human beings are capable of having? Discovering the state space of consciousness, i.e. the "map" of all states of consciousness, will be one of the most formidable tasks faced by consciousness science in the coming years (Berkovich-Ohana \& Glicksohn 2014; Emilsson 2020.) It is likely that we only explore a very narrow subset of the state space of consciousness in our lives (Pearce, 2020), though tools like psychedelics and meditation can significantly expand the range of conscious states that we experience. In this paper, we aim not to provide a scientific account of the state space of consciousness, but rather to test the effects of a series of audio samples on subjectively reported measures of consciousness. These samples include mindfulness meditations, "placebo highs," sound journeys, novel modes of introspection, and more (see Appendix for a synopsis of all the samples.)
The first iteration of the Museum of Consciousness premiered at Burning Man 2020. Sixteen artists contributed original work. Over 30 people attended the museum, and the showcase was nearly three hours long. A list of the exhibits, along with a very brief summary, was presented to the participants throughout the museum, and the attendees voted on the order in which they listened to the samples. Before each vote, the co-hosts queried the participants about their general mood, for example whether or not they felt the desire to be more embodied. After hearing the exhibits and practicing the techniques described within them, participants were then assigned to breakout rooms, where they had the chance to share their opinions with two other attendees. After leaving the breakout rooms, participants reflected on the samples in the main Zoom room. The discussion was generally freeflowing, though sometimes the co-hosts gave participants prompts like "describe your state of consciousness with just one word." Many of the 
participants were capable of discerning subtle changes that the samples engendered in their conscious experience, perhaps because of past experiences with altered states of consciousness. Several of the artists were present at the museum, so they were able to receive feedback directly from participants. Additionally, they were able to convey the context and significance of their artwork, as well as their creative process and any other details that the participants may have missed.

\section{ANALYSING SUBJECTIVE DATA ON CONSCIOUSNESS}

In order to quantify the effect of the museum on the participants' conscious experiences, we first needed to identify an appropriate model for measuring consciousness based on the self-reports of the participants. We decided on a recent sixdimensional model of altered self-consciousness that was inspired by meditation and psychedelics (Milliere et al, 2018). The model suggests that selfconsciousness can be broken down into six components:

(1) body ownership (is this body my own?);

(2) bodily awareness;

(3) self-location (where am I located in space?); (

4) phenomenal richness;

(5) access to semantic autobiographical information; and

(6) frequency of self-related thought.

We considered other scales of consciousness like 5Dimensional Altered States of Consciousness (5DASC) (Studerus et al. 2010), Mystical Experiences Questionnaire (MEQ) (Pahnke 1969), and Hood's Mysticism Scale (Hood 1975), all of which have been used to evaluate the phenomenology of meditation and the psychedelic state (Barrett \& Griffiths 2018; Liechti et al. 2017; Maclean et al. 2012). We chose Milliere's model because we anticipated that it would be a better fit for the exhibits currently in the museum. For example, one of the criteria in the 5D-ASC is "visionary restructuralization," which includes hallucinations and illusions, but it is unlikely that any of the samples would induce these visual phenomena without the aid of a psychoactive substance. The MEQ is bettersuited for intense, spiritual experiences that are brought on, for instance, by apparent encounters with God and powerful psychedelics since one of its components is transcendence of time and space. Hood's mysticism scale also contains a similar factor, namely the temporal/spatial quality of the experience, which is unlikely to be affected dramatically by the audio samples. Additionally, the scale is rather vague, consisting of characteristics like "unifying quality" and "inner subjective quality" that are rather difficult to evaluate.
We found that we needed to slightly adjust Milliere's model, since one of its factors, "bodily ownership," did not explain much of the variation in our conscious experience after listening to the samples. In other words, the samples did not modulate the extent to which we felt ownership over our own bodies. We replaced "bodily ownership" with "expansiveness," a general characteristic that refers to a relaxation of interpersonal boundaries (e.g. the boundary between self and other), the sense of a fixed self, the "rigidness" of thoughts and emotions, and more.

In our own assessment of each sample, we assign a numerical score from 1 to 5 for each component of the model. So, for example, if we thought that a particular sample strongly affects bodily awareness, then it would be assigned a score of 5 . It is notoriously difficult to rigorously evaluate subjective measures like the ones in Milliere's model, so we employed a technique called an elicitation method, which is designed to investigate "experiential microdynamics" - subtle, moment-to-moment variations in attention and emotion that usually go unnoticed without close introspection (Petitmengin \& Lachaux 2013). In the elicitation method, interviewers ask interviewees to describe their conscious experience with increasing precision; for instance, if the interviewee feels a sensation in his chest, then the interviewer asks how the sensation feels, how the sensation spreads through his body, how the sensation affects each region of his body, and so on. Surprisingly, the elicitation method has enabled epileptic patients to anticipate seizures by noticing the fine-grained changes that they bring about prior to their onset (Petitmengin et al., 2006). Due to limited time and resources, only one of the co-authors, K. Shinozuka, was able to conduct the elicitation method, so he interviewed himself.

\section{STRUCTURE OF THE MUSEUM}

Since the exhibits in the museum are audio-only, we wanted to think of a creative, audio-based method for creating recipes for consciousness augmention, as well as the interactive component of the museum. To fulfill these objectives, we decided to translate the numerical scores for each sample into musical scores. That is, we associate each dimension of the model with a musical note. The numerical score for that dimension corresponds to the number of times that the note is played in the musical score. So, for example, if a particular sample numerically scores a 5 on bodily awareness, a 3 on self-location, and a 2 on phenomenal richness, and bodily awareness corresponds to the musical note $\mathrm{C}$, self-location to $F$, and phenomenal richness to $G$, then the sample will be associated with a musical score consisting of 5 C's, 3 F's, and 2 G's. Additionally, the notes do not 
have equal duration, volume, and so on; we varied the rhythm and dynamics of each musical score.

We create recipes for consciousness augmentation by assembling scores into meta-scores, or qualia symphonies. For example, the scores associated with three different samples might fit together into a coherent whole in the same way that the three movements of a sonata combine into a unified piece. So, if multiple scores (musically) harmonize with one another, then the states of consciousness elicited by the corresponding samples will also "harmonize" with one another, in the sense that they blend well together. This idea symbolizes the Symmetry Theory of Valence from the Qualia Research Institute, which predicts that consonance between brain waves underlies more pleasant states of consciousness (Emilsson 2017.; Johnson 2016). Note that we are not claiming that the museum provides empirical evidence for the Symmetry Theory of Valence; rather, the museum serves as a creative metaphor for the theory.

At the start of the exhibit, we tell the participants about the six musical notes and the six dimensions of the model. Their task is to figure out the correspondences between the musical notes and the dimensions of the model. The task effectively turns the museum into an interactive game. At the end of each sample, the participants hear the associated musical score and fill out a form in which they rank the sample along the six dimensions of the model. Once they have heard all of the samples, they guess the correspondences.

After hearing each sample, participants will have the opportunity to provide feedback to the artist. Therefore, the museum follows a tripartite, cyclical structure designed to act as an engine for creative regeneration between the artist and the audience, thereby empowering both in equal measure:

1. Transmission - where the artist transmits their artwork, i.e. their sample, to the audience.

2. Experience -where the audience integrates and spends time processing the artwork by practicing the techniques or following the instructions prescribed by it. For example, if the sample contains a mindfulness meditation, then the audience does the meditation.

3. Feedback - where the audience directly communicates their thoughts and feelings back to the artist. The audience will be able to give feedback through the same form in which they rank the samples according to the model.

\section{APPENDIX: SUMMARY OF SAMPLES}

(Note: most of these synopses are written by the artists

themselves.)

1. "Tarot: Narrative Consciousness" (Adam Malone). Tarot teaches us to view our lives in a narrative sense, to view the past and the present as a story in which we are the central protagonist. As the writers, directors and narrators of the story of who we are, we become capable of creating a personal mythology, and perhaps take part in a much broader story that will remain long after we are gone! The narrative perception allows us to co-create a more conscious future, to transform ourselves and the world in which we live!

2. "How to Catch a Thought" (Alexander Beiner). A simple game unlike any other. It is the easiest and the hardest game there is: catching a thought as it enters your mind. When we play the game, we notice something extraordinary.

3. "Ocean of Stars" (Denise Rowe). An entire universe of wisdom and prayer infuses every decision that we make on our paths through this life. In this exhibit, you will visualize these ancient spiritual guides as an ocean of stars.

4. "Awakened Inquiry" (Heather Hargraves). Awakened Inquiry is a mixture of mindfulness, parts work and neuro linguistic processing. It is a simple method for moving into a core understanding of the deeper meaning and insights underlying the patterns of our ego and personality. This short-guided instruction can offer insight, creativity and guidance when we are feeling confused or stuck with aspects of our behaviour and emotional patterns. Close your eyes and open yourself to the wisdom contained within our embodied awareness.

5. "Placeebar" (Jay Cousins). Get high with nothing more than the power of your mind. You will make laughing gas out of the air that you breathe and make mind-altering cocktails with ingredients in your kitchen.

6. "You Are the Space in which You Live" (Kate Sagovsky). Space is the invisible sea that surrounds us, and in which we exist all the time. We don't often realise it but space is a huge part of what connects and defines us. This playful exploration allows you to start asking the question: how does my unconscious use of personal space define my experience of the world, and the world's experience of me?

7. "Embodied Double Consciousness" (Kevin Davidson). Explore a layering of awareness through gentle physical activities which are grounded in sensorial imagination.

8. "Third Eye Contact / Fourth Eye Contact" (Ad $\mathrm{Hoc}$ ). Using nothing more than a mirror, you will witness your third and fourth eye: the eternal, 
abiding witness that simply observes your entire conscious experience, with no judgment.

9. "Unfolding into Theta" (Robyn Landau). Theta acts as the gateway to your subconscious, holding sacred the depths of healing. Unfolding the layers and opening space to listen awakens the mind and body to restore, unlock and release. Drop deeply past the conscious layers, into the center of your natural healing source, connecting with the depths of knowledge accessed only in through a connection to your personal self experience.

10. "Ctrl+alt+float" (Sam Treasure). I have combined three techniques that have led to my most success with OBEs into "ctrl+alt+float": a variation of a Stephen LaBerge lucid dreaming technique, a technique "learned" during a false awakening, and a variation of the Spiegel eye roll. This labyrinthine combination confuses, resets and displaces the practitioner, making it easier to reach immersive out-of-body experiences.

11. "Dream Incubation" (Sarah Janes). A session of guided hypnagogia, sleep hypnosis and dream incubation - with the goal of enabling the participant to become more consciously aware of the journey one takes through the hypnagogic sleep borderland into REM/dream sleep. This technique looks to help you retain selfawareness throughout the process of very early dream content creation and hypnagogic imagery. Participants are required to lie down on their backs and relax, follow the sound of my voice and practice looking into the dream space. They will learn how to expand and deepen the experience this imagery and any audio hallucinations into a dream proper.

12. "The Bloom Smile" (Sofi LH). Naturally spread feelings of bliss through your body, once with movement and again with proprioception.

13. "Water Consciousness" (Zandra H). The water remembers everything, so it is important to explore different ways to communicate with our inner waters. In this exhibit, you will explore a simple but powerful practice using the breath to create a delicious cycle representing the waves of the ocean. With this practice you can also achieve a deep orgasmic sensation by living this experience as yours.

\section{REFERENCES}

Barrett, F. S., \& Griffiths, R. R. (2018) Classic Hallucinogens and Mystical Experiences: Phenomenology and Neural Correlates. Current Topics in Behavioral Neurosciences, 36, pp. 393-430. https://doi.org/10.1007/7854_2017_474
Berkovich-Ohana, A., \& Glicksohn, J. (2014) The consciousness state space (CSS)—A unifying model for consciousness and self. Frontiers in Psychology, 5. https://doi.org/10.3389/fpsyg.2014.00341

Emilsson, A.G. (2020) A Big State-Space of Consciousness. Qualia Computing. https://qualiacomputing.com/2020/01/18/a-big-statespace-of-consciousness/

Emilsson, A. G. (2017) Quantifying Bliss: Talk Summary $19 . \quad$ URL: https://static1.squarespace.com/static/5d72e78ebe27 7f3ec4400092/t/5f70fac535ffa16c0e867511/16012397 65985/Quantifying+Bliss.pdf

Hood, R. W. (1975). The Construction and Preliminary Validation of a Measure of Reported Mystical Experience. https://doi.org/10.2307/1384454

Johnson, M. E. (2016). Principia Qualia: Blueprint for a new science 84. CreateSpace

Liechti, M. E., Dolder, P. C., \& Schmid, Y. (2017). Alterations of consciousness and mystical-type experiences after acute LSD in humans. Psychopharmacology, 234(9), pp. 1499-1510. https://doi.org/10.1007/s00213-016-4453-0

Maclean, K. A., Leoutsakos, J.-M. S., Johnson, M. W., \& Griffiths, R. R. (2012). Factor Analysis of the Mystical Experience Questionnaire: A Study of Experiences Occasioned by the Hallucinogen Psilocybin. Journal for the Scientific Study of Religion, 51(4), pp. 721-737.

Pahnke, W. N. (1969) Psychedelic drugs and mystical experience. International Psychiatry Clinics, 5(4), pp. 149-162.

Pearce, D. (2020) The imperative to abolish suffering: An interview with David Pearce - Sentience Research. https://sentience-research.org/the-imperative-toabolish-suffering-an-interview-with-david-pearce/ (Retrieved March 17, 2021)

Petitmengin, C., Baulac, M., \& Navarro, V. (2006) Seizure anticipation: Are neurophenomenological approaches able to detect preictal symptoms? Epilepsy \& Behavior, 9(2), pp. 298-306. https://doi.org/10.1016/j.yebeh.2006.05.013

Petitmengin, C., \& Lachaux, J.-P. (2013) Microcognitive science: Bridging experiential and neuronal microdynamics. Frontiers in Human $\begin{array}{llll}\text { Neuroscience, } & 7, & \text { p. }\end{array}$ https://doi.org/10.3389/fnhum.2013.00617

Studerus, E., Gamma, A., \& Vollenweider, F. X. (2010) Psychometric Evaluation of the Altered States of Consciousness Rating Scale (OAV). PLoS ONE, 5(8). https://doi.org/10.1371/journal.pone.0012412 